\title{
THE DYNAMICAL EVOLUTION OF A TUBULAR LEONID PERSISTENT TRAIN
}

\author{
PETER JENNISKENS AND DAVID NUGENT \\ SETI Institute, NASA Ames Research Center, Mail Stop 239-4, Moffett Field, CA 94035 \\ E-mail:pjenniskens@mail.arc.nasa.gov \\ and \\ JOHN M.C. PLANE \\ School of Environmental Sciences, University of East Anglia, Norwich NR4 7TJ, UK \\ E-mail: J.Plane@uea.ac.uk
}

(Received 26 May 2000; Accepted 16 August 2000)

\begin{abstract}
The dynamical evolution of the persistent train of a bright Leonid meteor was examined for evidence of the source of the luminosity and the physical conditions in the meteor path. The train consisted of two parallel somewhat diffuse luminous tracks, interpreted as the walls of a tube. A general lack of wind shear along the trail allowed these structures to remain intact for nearly $200 \mathrm{~s}$, from which it was possible to determine that the tubular structure expanded at a near constant $10.5 \mathrm{~ms}^{-1}$, independent of altitude between 86 and $97 \mathrm{~km}$. An initial fast decrease of train intensity below 90 $\mathrm{km}$ was followed by an increase in intensity and then a gradual decrease at longer times, whereas at high altitudes the integrated intensity was nearly constant with time. These results are compared to a model that describes the dynamical evolution of the train by diffusion, following an initial rapid expansion of the hot gaseous trail behind the meteoroid. The train luminosity is produced by $\mathrm{O}$ ('S) emission at $557 \mathrm{~nm}$, driven by elevated atomic $O$ levels produced by the meteor impact, as well as chemiluminescent reactions of the ablated metals $\mathrm{Na}$ and $\mathrm{Fe}$ with $\mathrm{O}_{3}$. Ozone is rapidly removed within the train, both by thermal decomposition and catalytic destruction by the metallic species. Hence, the brightest emission occurs at the edge of the train between outwardly diffusing metallic species and inwardly diffusing $\mathrm{O}_{3}$. Although the model is able to account plausibly for a number of characteristic features of the train evolution, significant discrepancies remain that cannot easily be resolved.
\end{abstract}

Keywords: Airglow, chemistry, dynamics, Leonids 1998, lower thermosphere, mesosphere, meteor, persistent train 


\section{Observations}

At 01:31:16 UT on November 17, 1998, a bright Leonid meteor (Figure 1) erupted over the southern U.K., moving from Southend-on-Sea to just beyond Reading. Amateur asstronomer Sandy Osborough, from Chippenham, Wiltshire $\left(51^{\circ} 28 \mathrm{~N}, 02^{\circ} 07^{\prime} \mathrm{W}\right)$, was located near the end point of the trajectory. The meteor was outside the field of view of his intensified video camera, but the scattered light in the atmosphere left a flash in the video record. Osborough adjusted the viewing direction of the camera and obtained a particularly nice record of the "Chippenham" persistent train between 15 seconds and $2 \mathrm{~m} 54 \mathrm{~s}$ after the flash (Figure 2, right part of each frame). The train persisted longer than that, but the camera was pointed elsewhere. He used a high quality intensified video camera, and the close range of the train $(116-80 \mathrm{~km})$, produced a spatial resolution of $0.2 \mathrm{~km} / \mathrm{pixel}$.

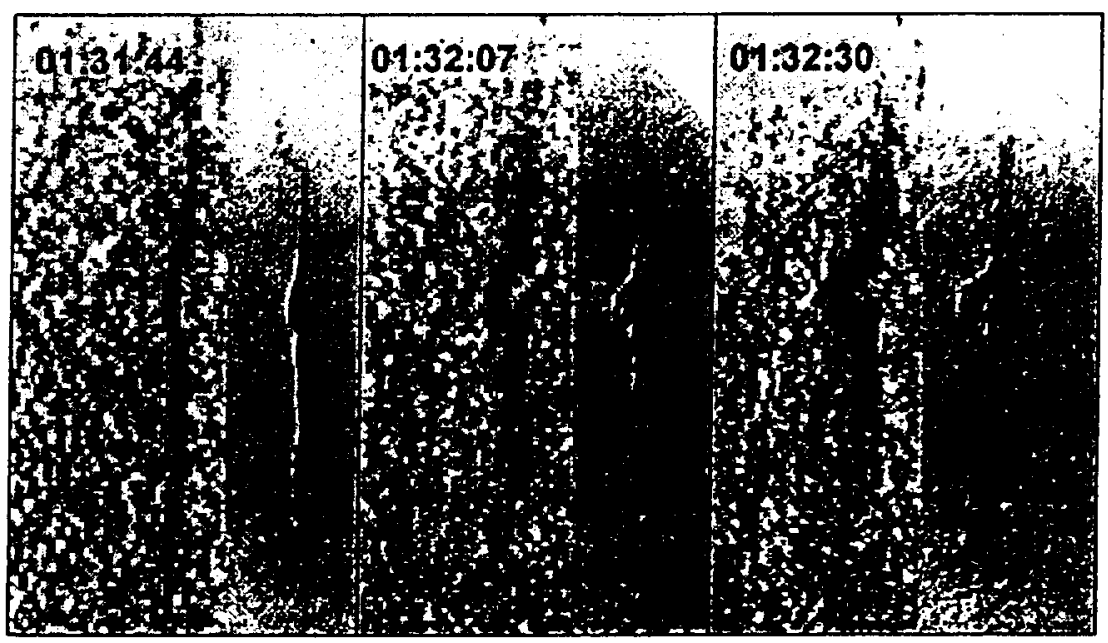

Figure 2. The train as seen by Tim Haymes from the perspective of Knowl Hill (left) and by Sandy Osborough from Chippenham (right).

The train was also filmed by Tim Haymes from Knowl Hill, Berks. $\left(00^{\circ} 48^{\prime} 51.3^{\prime \prime} \mathrm{W}, 51^{\circ} 30^{\prime} 22.1^{\prime \prime} \mathrm{N}\right)$, again from relatively short range $(\sim 102 \mathrm{~km})$. Haymes used a $28 \mathrm{~mm} \mathrm{f} / 2$ lens imaging onto an $18 \mathrm{~mm} 2^{\text {pd }}$ generation $\mathrm{MCP}$ image intensifier ( $30 \mathrm{lpi}$ ). The image resolution was also $0.2 \mathrm{~km} / \mathrm{pixel}$, but the noisier tube created a less exceptional image. 


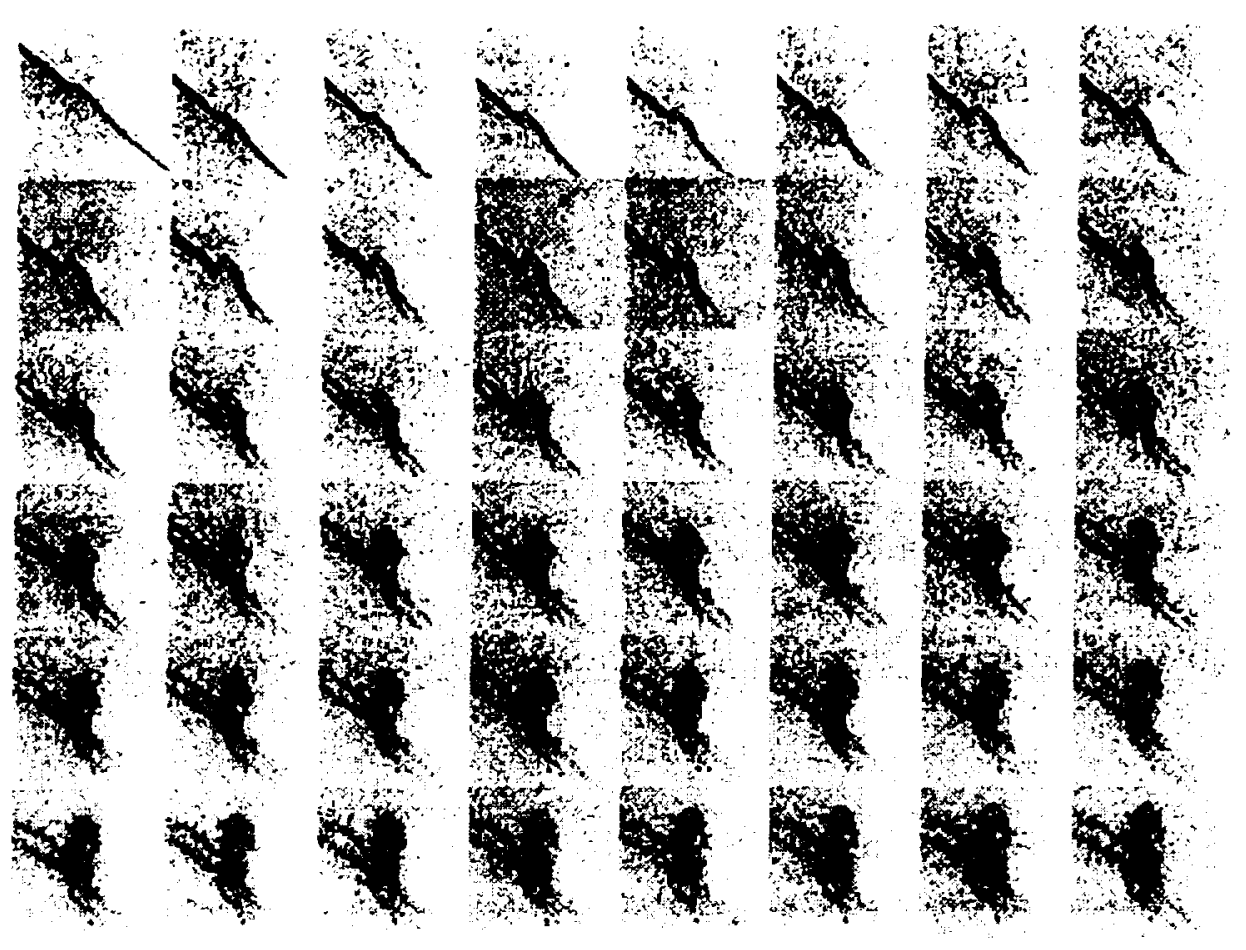

Figure 3. Sequence of images as seen from Chippenham between $0 \mathrm{~m} 15 \mathrm{~s}$ and $02 \mathrm{~m} 52 \mathrm{~s}$ after the meteor, showing the formation of the loop and the evolution of the linear parts.

This train is very unusual because the two straight sections of the trajectory remain almost straight with respect to the star background (Figure 3). Note that the lower part gradually gains upon the higher part and may be rising in altitude. Only the bright middle section forms a loop, which eventually overlaps in the line of sight. At the position of the loop, the wind direction changes dramatically with altitude. It is from the North in the straight sections, while from the East in the distorted middle part and end sections, with strong wind shears in the transition regions. The magnitude of the wind vector changes only by a factor of $2-3$. Most of the wind shear is laminar, preserving the tubular structure of the train during distortion.

The morphology of the train is either that of a tube, where the two trails represent the longest line of sight along the walls of the tube, or they represent the turbulent top and bottom of a ribbon-like structure. The most likely morphology is that of a tube, for two reasons. First of 


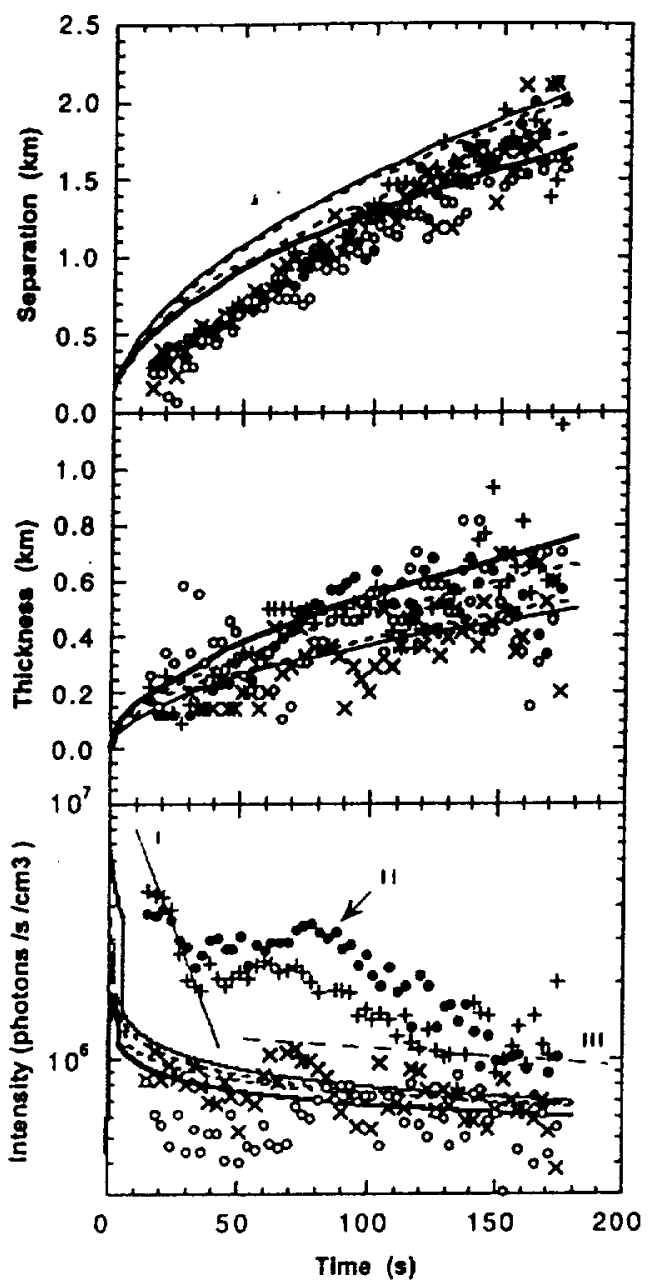

Figure 5. (a) The separation between the peak intensity of the tubular walls; (b) the thickness of the tubular wall; and (c) the integrated intensity of the tubular walls as a function of time. Symbols are: Pos. I ( $)$, Pos. II (t), Pos. III (x), Pos. IV (o). Model results shown are for 86 (solid line), 89 (dashed), 95 (dashed) and $97 \mathrm{~km}$ altitude (thick solid line).

The tubular walls are resolved at the end of the exposure. We measured the thickness at half peak intensity as a function of time and deconvolved with a 2-pixel wide Gaussian response curve. We find that the turbulent walls tend to show slightly more billowing over time, gradually thickening, but the expansion slows down after about $100 \mathrm{~s}$ (Figure 5b). 
6). The brightness decays fastest at the lower altitude end of the train (left in Figure 6), while the higher altitudes follow in succession.

Phase (II) is characterized by the initial brightening of the train. The intensity peaks earliest towards the middle part of the trajectory, with mechanisms delaying the increase at very high and very low altitudes. The subsequent decay has a time constant of about $63 \pm 2 \mathrm{~s}$ at $86 \mathrm{~km}, 70$ $\pm 2 \mathrm{~s}$ at $89 \mathrm{~km}, 150 \pm 20 \mathrm{~s}$ at $95 \mathrm{~km}$, and about $190 \mathrm{~s}$ at $97 \mathrm{~km}$ altitude.

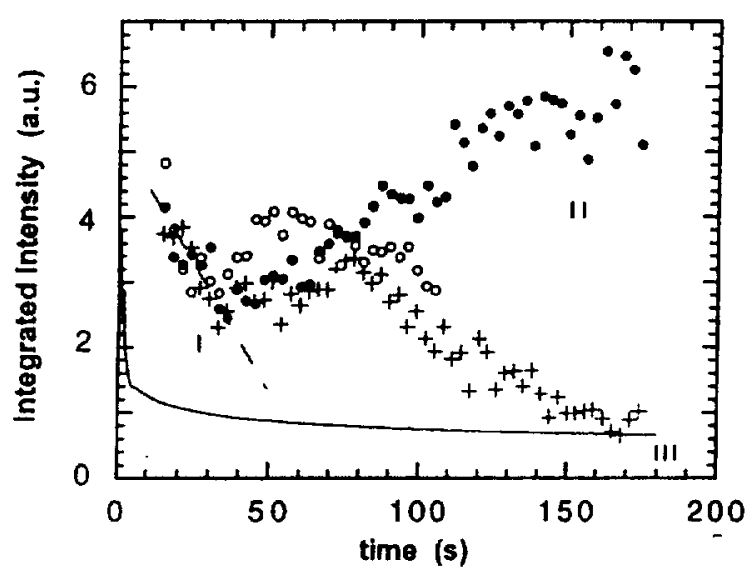

Figure 7. Integrated intensity variation of the knot $(\cdot)$ and the total intensity of the loop (o), scaled to that of the "quiet" train at position I (+). Solid line shows a model fit for the height of $86 \mathrm{~km}$ (in units of $10^{6}$ photons $\mathrm{cm}^{-3} \mathrm{~s}^{-1}$ ).

This intensity increase during Phase (II) may be related to the brightening phenomenon that enhances the brightness of the loop between 90 and $94 \mathrm{~km}$. The integrated intensity of the loop (box in Figure 4) is shown in Figure 7, in relation to the brightness of the linear part of the train at position II. At the end of the observation, the loop is the only part that remains clearly visible. A remarkable feature is the "knot" (marked in Figure 4), where wind shear appears to be particularly high at the beginning of the observations. We see that the tubular structure breaks down during bending and a wall of billowing emission is observed. Here, we find the quickest brightness increase, which is followed by a decrease that mimics the linear part of the train (open circles in Figure 7).

Phase (III) is most apparent in the high altitude part of the train, but also visible in position II. This phase represents the late stages of train evolution. It is well represented by our model calculations below. 
produce additional $O$ (the green tail of $L$. caused by emission from $O\left({ }^{\prime} S\right)$ which is : O concentration, as discussed below). ? both from thermal dissociation in the ini catalysed destruction. Effective catalyti on the concentration of metallic species $r$ all neutral metal atoms, as well as metalli. alkali metal ions, participate in cataly: Helmer, 1994). id meteors, in particular, is nly dependent on the atomic depletion of $\mathrm{O}_{3}$ could arise $y$ very hot train, and metalmoval places a lower limit aced by ablation. Note that is with the exception of the $o_{3}$ destruction (Plane and

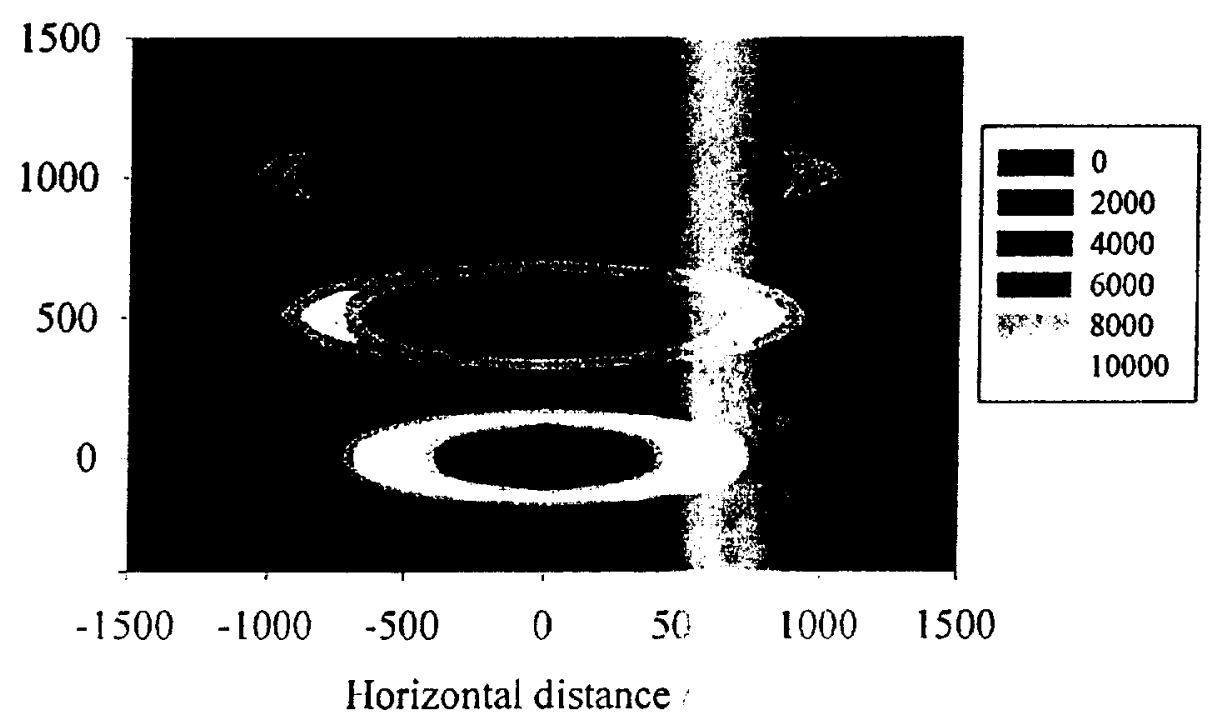

Figure 8. Three cross-sections through the pe: $\mathrm{km}$, showing the modeled emission intensity : 150 s after the meteor. The 100 and $150 \mathrm{~s}$ secti. by 500 and $1000 \mathrm{~m}$, respectively, for the purp emission patch visible at $50 \mathrm{~s}$ and $100 \mathrm{~s}$ is due $\mathrm{t}$. due to chemiluminescence from metal atom reac:

at train at an altitude of 86 $\therefore$ 50s (bottom), 100s and isve been displaced upward of presentation. The central i) emission, the outer ring is with ambient ozone.

We have therefore constructed a model of a conid meteor train in order to simulate the train expansion, the increa: in wall thickness and the observed brightness variation as a function position and time. The model makes the following assumptions:

1. The meteoroid ablates according to deceleration equation (particle density $=3,200 \mathrm{~kg} \mathrm{~m}^{-3}$, drag : fficient $=0.75$ and shape 
6. The "green line" emission at $557 \mathrm{~nm}$ from the $\mathrm{O}\left({ }^{1} \mathrm{~S}-{ }^{1} \mathrm{D}\right)$ transition (termed [OI]) was assumed to be produced by the Barth mechanism with the absolute intensity calculated using the parameterisation of Murtagh et al. (1990). Assuming that this emission dominates the train emission immediately after the meteor, then in order to simulate the observed decrease in intensity of the Chippenham train at longer times the model requires that about $15 \%$ of the $\mathrm{O}_{2}$ in the initial train was dissociated.

The train model was then run with a spatial resolution of 25 meter and integration time-step of 0.2 seconds. Figure 8 shows cross sections through the modelled train at an altitude of $86 \mathrm{~km}$ at $50 \mathrm{~s}, 100 \mathrm{~s}$ and $150 \mathrm{~s}$ after the meteor. Initially, strong [OI] emission is observed at the center of the trail because of the enhanced $\mathrm{O}$ atom concentration produced from dissocation of atmospheric $\mathrm{O}_{2}$ by the meteor. The [OI] intensity is dependent on $[\mathrm{O}]^{3}$, so that the intensity falls very quickly as the atomic $O$ diffuses outwards from the centre of the train.

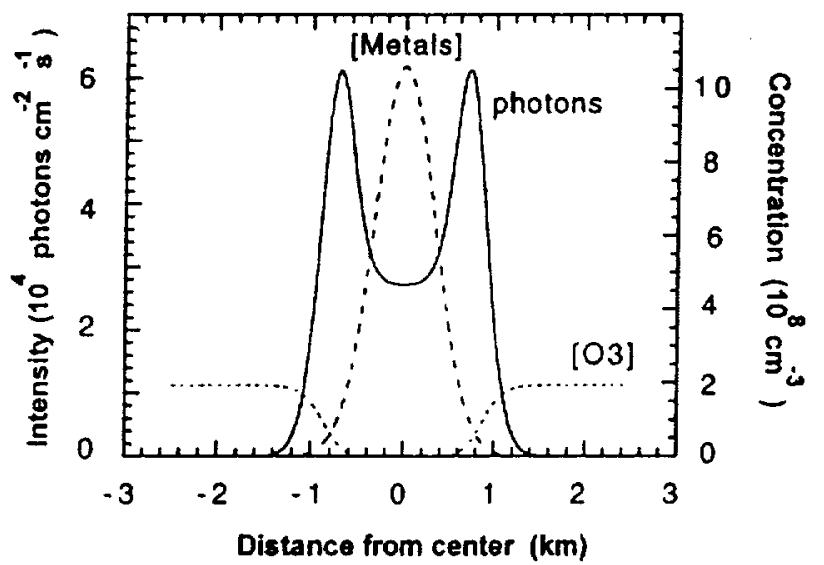

Figure 9. Variation in the metal atom and $\mathrm{O}_{3}$ densities across the center of the trail at $98 \mathrm{~km}, 100 \mathrm{~s}$ after the meteor. The vertically integrated emission intensity which would be observed from the ground, is shown for comparison.

As shown in Figure 9, after $100 \mathrm{~s}$ the $\mathrm{O}_{3}$ concentration within the train has been reduced by orders of magnitude by the combined effects of thermal decomposition and catalytic destruction. The concentration profile of the metallic species is approximately Gaussian, as expected for diffusion-controlled transport. The metallic emission is strongest at the edge of the train, where fresh ambient $\mathrm{O}_{3}$ is diffusing inwards. 
between the central minimum and the wall maxima. In fact, the lack of contrast in the model is all the more striking because we have maximised the contrast by using a smaller vertical diffusion coefficient to reduce the vertical transport of metallic species and fresh $\mathrm{O}_{3}$, thereby minimising the wall brightness in the top and bottom parts of the tube (Figure 8). The lack of contrast is not explained by decreasing the initial ozone concentration in the center faster, for example as a result of photodissociation of ozone by the meteoric UV light (Zinn et al., 1999).

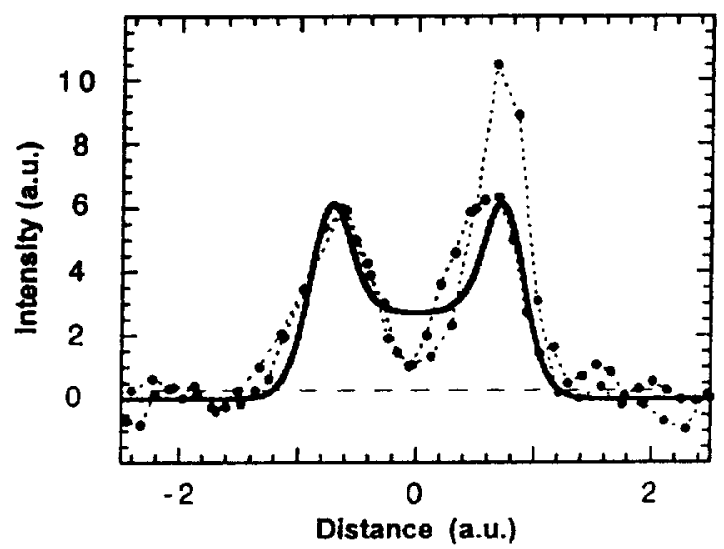

Figure 10. Contrast in brightness of center and walls in model (solid line) and observations. Model in units of $10^{4}$ photons $\mathrm{cm}^{-3} \mathrm{~s}^{-1}$.

Also, there are significant difficulties with modeling the brightness behavior (Figure $5 \mathrm{c}$ ). The phase I decay is thought to be due to emission of the forbidden $557 \mathrm{~nm} \mathrm{O}$ ( ${ }^{1} S$ - 'D) transition. Indeed, meteors of lesser brightness are known to have persistent emission on a time scale of about 10 seconds, sometimes referred to as the meteor "wake" (Halliday, 1958). This is a direct result of molecular oxygen dissociation by the meteor and has been well recorded in photographic and TV video spectra (e.g. Borovicka et al., 1996). Figure 11 reproduces one of our own measurements, where it can be seen that the [OI] emission starts shortly after the meteor itself, peaks, and then rapidly decays. The model predicts that the [OI] emission in the Chippenham train would have been brighter at and below $90 \mathrm{~km}$ (positions I and II), since this is where more atomic $\mathrm{O}$ is produced in the meteor. The emission is predicted to decay on a time scale of only about $10 \mathrm{~s}$ (due to its $[\mathrm{O}]^{3}$ dependence and the rapid outward diffusion of atomic $O$ ), rather than the observed $30-40 \mathrm{~s}$ in phase $\mathrm{I}$. Note also that the [OI] line intensity should be concentrated 
increase the interfacial area between the train and surrounding air, enhancing the rate of chemiluminescent reactions between metallic species and $\mathrm{O}_{3}$. If such turbulence spread horizontally rather than vertically, this would help to explain the high contrast between train center and walls.

The model assumes that the longer-lived emission is due to metallic atoms reacting with $\mathrm{O}_{3}$. A rapid decline in train intensity, such as observed in phase II at 86 and $89 \mathrm{~km}$, could be because of depletion of these metals. However, there are no reactions with background atmospheric constituents such as $\mathrm{H}_{2} \mathrm{O}, \mathrm{CO}_{2}, \mathrm{O}_{2}$ etc. that will convert these species to stable forms on the time scale observed. The only other possibility is that these metals are reacting with the high concentration of silicates and other debris in the trail, although again the time scale of 200 seconds is very short.

In summary, the present model coupling meteor ablation with simple diffusive transport of the resulting train is able to account satisfactorily for some of the significant features of this unusual event. These include the appearance of two luminous tracks, the average rate of increase of their separation and thickness, and some aspects of the luminous emission decay. However, the model fails to explain the strikingly constant rate of separation increase, which cannot be diffusive in nature, and the complex variation of the emission with time at some altitudes, amongst others. Clearly, there is still much to be understood about the nature of persistent trains.

\section{Acknowledgments}

Amateur observers Sandy Osborough, Tim Hayes and Steve Evans are congratulated with their fine records of train and meteor. We are thankful for their kindness in making these records available for analysis. We also thank John Green and David Stephens at the University of East Anglia for helpful discussions on turbulent transport. This work forwards the goals of the Pro-Amat working group of IAU Commission 22. The work is supported by grants from NASA's Suborbital MITM and Exobiology programs, and by the NASA Advanced Missions and Technology program for Astrobiology. Editorial handling: Noah Brosch. 